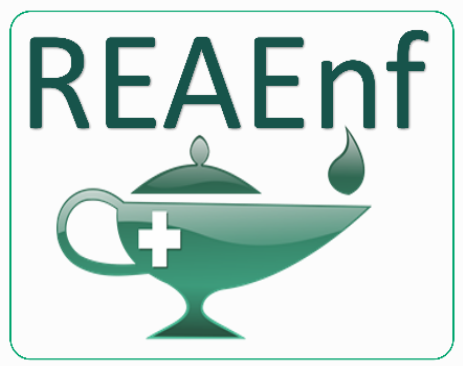

Revista Eletrônica Acervo Enfermagem
REVISÃO BIBLIOGRÁFICA

Recebido em: 6/2020

Aceito em: 7/2020

Publicado em: 11/2020

\title{
Transporte de pacientes críticos: a visão do enfermeiro
}

\author{
Transportation of critical patients: the nurse's view
}

Transporte de pacientes críticos: la visión de lo enfermeiro

José Petrucio da Silva Filho ${ }^{1 *}$, Rosane Pereira dos Reis ${ }^{1}$, Douglas Ferreira Rocha Barbosa ${ }^{1}$, Adriana Leal Lima ${ }^{1}$, Marcelle Perdigão Gomes ${ }^{1}$, Daniele Gonçalves Bezerra ${ }^{2}$, Layanne Ramalho Jacob $^{3}$, Alaine dos Santos Silva Martins ${ }^{3}$, Janieide Ferreira da Silva ${ }^{3}$, Michelâyne Raniely dos Santos ${ }^{3}$.

Resumo: O objetivo desse estudo é descrever a visão do enfermeiro frente ao transporte de pacientes crítico. Trata-se de uma revisão integrativa, em que foram consultadas junto à Biblioteca Virtual de Saúde (BVS) acessando as bases de dados: Medical Literatura Latino-Americana e do Caribe em Ciências da Saúde (LILACS) e Base de Dados em Enfermagem (BDENF), totalizando 11 artigos. Para que aconteça um transporte garantido, é indispensável um bom planejamento, uma equipe habilitada e, quando necessário for, o uso de equipamentos confiáveis, para submissão de um paciente ao transporte. Neste estudo ficou claro que em virtude da variedade da área de intervenções e fatores que intervêm e, em alguns casos, limitam as intervenções, o transporte do paciente crítico é uma circunstância complicada que envolve aspectos proeminentes, especialmente no que se refere à atuação dos profissionais durante o seu processo, percebe-se que ações de melhorias para um transporte seguro de todos os pacientes críticos precisam ser criadas, padronizando ações e processos através de protocolos para que todos os profissionais sigam a mesma linha de cuidado.

Palavras-chave: Transporte de pacientes, Cuidados críticos, Cuidados de enfermagem.

\begin{abstract}
The objective of this study is to describe the nurse's opinion on the critical transport of patients. This is an integrative review, in which they were consulted with the Virtual Health Library (VHL) accessing the databases: Latin American and Caribbean Medical Literature in Health Sciences (LILACS) and Nursing Database (BDENF), totaling 11 articles. For guaranteed transport to happen, good planning, qualified equipment and, when necessary, the use of reliable equipment are essential to send a patient to transport. In this study it was clear that due to the variety of the area of interventions and factors that intervene and, in some cases, limit the interventions, the transport of the critical patient is a complicated circumstance that involves prominent aspects, especially with regard to the performance of professionals. During your process, it is observed that it is necessary to create improvement actions for the safe transportation of all critically ill patients, standardizing actions and processes through protocols so that all professionals follow the same line of care.
\end{abstract}

Keywords: Transport of patients, Critical care, Nursing care.

\footnotetext{
${ }^{1}$ Faculdade Estácio de Alagoas, Maceió - AL. *E-mail: ensinoepesquisa7@outlook.com

2 Universidade Federal de Alagoas (UFAL), Maceió - AL.

${ }^{3}$ Universidade Estadual de Ciências da Saúde de Alagoas, Maceió - AL.
} 
Resumen: El objetivo de este estudio es describir la opinión de la enfermera sobre el transporte crítico de pacientes. Esta es una revisión integradora, en la cual fueron consultados con la Biblioteca Virtual en Salud (BVS) accediendo a las bases de datos: Literatura Médica Latinoamericana y del Caribe en Ciencias de la Salud (LILACS) y Base de Datos en Enfermería (BDENF), totalizando 11 artículos. Para que el transporte garantizado suceda, una buena planificación, un equipo calificado y, cuando sea necesario, el uso de equipos confiables son esenciales para enviar a un paciente al transporte. En este estudio quedó claro que debido a la variedad del área de intervenciones y factores que intervienen y, en algunos casos, limitan las intervenciones, el transporte del paciente crítico es una circunstancia complicada que involucra aspectos prominentes, especialmente con respecto al desempeño de los profesionales. durante tu proceso, se observa que es necesario crear acciones de mejora para el transporte seguro de todos los pacientes críticos, estandarizando acciones y procesos a través de protocolos para que todos los profesionales sigan la misma línea de atención.

Palabras clave: Transporte de pacientes, Cuidado crítico, Atención de enfermería.

\section{INTRODUÇÃO}

Atualmente o período em que se vive num mundo abalado por alterações constantes e marcantes, onde o cuidar das pessoas é cada vez mais fundamentado na ciência, a enfermagem vem como uma profissão que está cada vez mais envolvida com a preparação de um corpo científico de conhecimentos relacionados à sua prática, para então oferecer um cuidado ainda mais de qualidade e humano (FRANÇOLIN L, et al., 2012).

Segundo Pedreira LC, et al. (2013), esta profissão registrou nos últimos anos um enriquecimento, em nível de formação, bem como no que diz respeito à complexificação e dignificação do seu exercício profissional, valorizando as competências dos enfermeiros na comunidade científica, assim como a qualidade e eficácia da prestação de cuidados. A questão do transporte de pacientes críticos, tem instituído uma causa de debate e conferência por parte dos profissionais de saúde ao nível nacional, tendo como fundamental interlocutor a Sociedade Portuguesa de Cuidados Intensivos (SPCI).

É considerado paciente critico aquele que apresenta sinais de iminência à sua vida e/ou seu bem estar e apresenta quebra densa de um ou mais órgãos, a sua sobrevivência depende de meios avançados de monitorizarão e tratamento específico. No entanto, o enfermeiro que cuida do paciente crítico vê a unidade como um ambiente de cuidados críticos como um sinal de morte iminente. O transporte do paciente crítico é a expansão da unidade de origem, deste modo precisa ser realizado de forma segura, evitando assim o agravamento do estado clínico (MORAIS SA e ALMEIDA LF, 2013).

De acordo com Andrade PB, et al. (2012), a decisão de transportar um paciente crítico, necessita de uma fundamentada avaliação e critérios dos melhoramentos e seus riscos. Um dos motivos principais para transportar o paciente crítico é a necessidade dos cuidados adicionais (tecnologia e especialistas) não disponíveis no lugar onde o paciente é encontrado. O transporte pode ser tanto intra como inter-hospitalar, o transporte intra-hospitalar é considerado fundamental para a realização dos testes de diagnósticos como a tomografia computadorizada, a ressonância nuclear magnética, a angiografias, dentre outros para intervenções terapêuticas como para o centro cirúrgico, ou para internação em centro de terapia intensiva (CTI). O transporte inter-hospitalar é realizado sempre que se precisa de maiores recursos humanos, análises, tratamento e de suporte avançado de vida, que não estão presentes no hospital de origem, e o mesmo pode ser realizado por via terrestre ou por via aérea.

O transporte de pacientes críticos, envolve 3 etapas: a decisão da transferência (é um ato médico e pressupõe que houve avaliação de benefícios e riscos intrínsecos ao transporte); o planejamento (precisa ser realizado pela equipe médica e de enfermagem do serviço); efetivação (fica a cargo da equipe de transporte selecionada) cuja responsabilidade só termina no momento da entrega do paciente à equipe do serviço destinatário (MENEGUIN S, et al., 2014). 
Cabe destacar que o transporte de pacientes críticos, que é a modalidade intra-hospitalar, está associado à realização de exames diagnósticos ou terapêuticos em pacientes críticos e requer a substituição de aparelhamentos de suporte, de monitorizarão, além da continuação da infusão de medicamentos e transferência para maca de transporte. O transporte intra-hospitalar é um momento de instabilidade e riscos para o paciente, com possibilidade de intercorrências relacionadas à falhas técnicas, alterações fisiológicas do paciente, tempo de transporte, bem como a equipe que o realiza (ALMEIDA AC, et al., 2012).

Segundo Ong MS e Coiera E (2010), a preocupação com o transporte do paciente crítico tem surgido de forma sólida na última década, uma vez que, o sucesso no transporte intra- hospitalar necessita conjuntamente do planejamento e também da atuação organizada pela equipe de saúde e como também da escolha de equipamentos indicados. Assim, um aspecto importante no transporte do paciente é o diálogo prévio de todas as informações indispensáveis entre a equipe que transporta o paciente e a que irá recebêlo, na qual não seja comprometida sua segurança e a sequência dos cuidados de saúde seja melhor reforçada.

Pedreira LC, et al. (2014), traz a compreensão de que o transporte do paciente crítico necessita de uma tática cuidadosa principalmente quanto à comunicação com a equipe, identificando as informações fundamentais relacionadas ao paciente. Sendo um dos grandes empecilhos marcados pela equipe de enfermagem, as barreiras na comunicação. Essas dificuldades seriam bem menores se existisse uma ampla relação dentre as equipes envolvidas com o transporte em um cenário sossegado. Salienta-se também que os profissionais envolvidos no descolamento do paciente quando não usado o tempo necessário para informações na passagem de plantão do paciente ou não sabem seu verdadeiro quadro clínico, dados importantes poderão ser completamente perdidos.

Neste contexto, as ações de enfermagem determinam frequentemente a recuperação e/ou agravamento clínico dos pacientes. O cuidado de enfermagem é uma ferramenta das ações guiadas por regras, rotinas ou normas que propõem uma observação proeminente e disciplinada. É importante prevenir agravos à saúde por meio das ações de enfermagem direcionadas. O papel do enfermeiro nessas ações de transporte do paciente crítico é interdisciplinar e integralizado quando se norteia na necessidade da saúde do paciente. Cabe salientar que a enfermagem vem acompanhando os avanços de tecnológicos na área hospitalar e com isso vem aperfeiçoando seus conhecimentos para realizar seus cuidados junto à população hospitalizada. No entanto, faz-se ainda necessário aperfeiçoar os cuidados de forma ampla, tendo em vista o controle do risco durante o transporte de pacientes, amortizando os agravos à saúde da população hospitalizada (FRANÇOLIN L, et al., 2012).

A partir deste contexto e diante do conhecimento sobre o assunto, pretende-se realizar um estudo de revisão integrativa da literatura, levantando a seguinte norteadora: Qual a visão do enfermeiro frente ao transporte de pacientes críticos? Sendo assim, o objetivo desta pesquisa é descrever a visão do enfermeiro frente ao transporte de pacientes críticos. Este estudo é relevante, pois ele almeja expandir os conhecimentos dos profissionais de saúde sobre a temática e servir como fonte de conhecimento para futuras pesquisas, uma vez que, é notório ressaltar que enfermeiros são elementos fundamentais para transferência de pacientes críticos (PEDREIRA LC, et al., 2013).

Este estudo tem como objeto a visão do enfermeiro frente ao transporte de pacientes críticos. $O$ interesse em estudar esse assunto surgiu a partir da vivência do pesquisador diante da trajetória profissional vivenciada no setor de transferência em um hospital público, onde foi possível observar o escasso conhecimento dos profissionais de enfermagem em relação à transferência de pacientes críticos, e com isso surgiu à motivação em aprofundar o conhecimento técnico e científico sobre esta temática.

\section{MÉTODOS}

Trata-se de uma revisão integrativa de literatura, tendo uma abordagem qualitativa. O levantamento bibliográfico desse estudo foi realizado no ano de 2019, através da consulta direta na internet através dos dados online disponíveis na Biblioteca Virtual de Saúde (BVS) e nas seguintes bases de dados Literatura 
Latino-Americana e do Caribe em Ciências da Saúde (LILACS), Scientific Eletronic Library Online (SciELO), e pela Base de Dados em Enfermagem (BDENF).

$\mathrm{Na}$ busca inicial foram considerados os títulos e os resumos dos artigos para a seleção ampla de prováveis trabalhos de interesse, sendo destacados os resumos (dos artigos que não tinham texto acessível) e os textos completos dos artigos, para a busca dos artigos foi utilizados os seguintes descritores em Ciências da Saúde (DeCS): transporte de pacientes, transferência de pacientes, cuidados críticos e enfermeiros.

Foi utilizado como critério de inclusão artigos na íntegra e que retratavam a temática abordada, publicados e indexados nos bancos de dados referidos, dos últimos 6 anos sendo de 2014 a 2019, na línguas portuguesa, inglesa e espanhola, e em caso de títulos repetidos, apenas um deles foi incluídos e os artigos disponíveis gratuitamente. Assim, também foram utilizadas referências localizadas nos artigos selecionados, pela sua importância do seu conteúdo para o estudo. Em relação aos critérios de exclusão, optou-se por não utilizar artigos que não correspondiam ao objeto de estudo, assim como também textos que se encontravam incompletos, textos duplicados e que não eram consideradas pesquisas e/ou artigos.

A seleção dos estudos foi realizada de forma independente, realizado o cruzamento dos descritores utilizando operador booleano AND sendo localizados 132 artigos. Por ser o único a mostrar uma busca precisa acerca do foco da temática, com isso sendo possível encontrar os artigos para a realização do estudo.

\section{RESULTADOS}

Através da aplicação dos critérios de inclusão resultou em 132 artigos, sendo que 121 foram excluídos por não atenderem o foco do trabalho resultando em uma amostra filtrada de 11 publicações (Tabela 1).

Tabela 1 - Distribuição das referências selecionadas de acordo com o ano em que foram publicadas. $(n=11)$.

\begin{tabular}{ccc}
\hline Ano & Referências Selecionadas & Porcentagem (\%) \\
\hline 2014 & 3 & 27,27 \\
2015 & 0 & 0 \\
2016 & 2 & 18,19 \\
2017 & 3 & 27,27 \\
2018 & 0 & 0 \\
2019 & 3 & 27,27 \\
\hline Total & 11 & $100 \%$ \\
\hline
\end{tabular}

Fonte: Silva Filho JP, et al., 2019.

Na tabela abaixo evidencia que na base de dados LILACS foi encontrado o maior número de referências, como também, referências selecionadas para a composição do corpo da pesquisa, logo após pela BDENF e pela SciELO (Tabela 2).

Tabela 2 - Distribuição das referências obtidas nas bases de dados LILACS, BDENF e SciELO. $(n=11)$.

\begin{tabular}{cccc}
\hline Bases de dados & Referências obtidas & $\begin{array}{c}\text { Referências } \\
\text { selecionadas }\end{array}$ & $\begin{array}{c}\text { Porcentagem total } \\
(\%)\end{array}$ \\
\hline LILACS & 86 & 5 & 45,46 \\
BDENF & 27 & 3 & 27,27 \\
SciELO & 19 & 3 & 27,27 \\
\hline Total & 132 & 11 & 100 \\
\hline
\end{tabular}

Fonte: Silva Filho JP, et al., 2019. 
No atual estudo, onze artigos atenderam rigorosamente à seleção da amostra previamente estabelecida. Abaixo são expostos os artigos selecionados em ordem cronológica de publicação, observar-se assim uma densa diversidade de periódicos que publicaram artigos sobre a temática estudada (Tabela 3).

Tabela 3 - Artigos científicos selecionados nas bases de dados LILACS, SCIELO e BDENF segundo o título, periódico e ano.

\begin{tabular}{cccc}
\hline Bases de dados & N & Título do artigo & Periódico/ Ano \\
\hline 1 & $\begin{array}{c}\text { Transporte intra/extra-hospitalar de } \\
\text { crianças: implicações da equipe de } \\
\text { Enfermagem. }\end{array}$ & $\begin{array}{c}\text { Revista Espaço para a } \\
\text { Saúde/ 2019 }\end{array}$ & Journal of Nursing UFPE/ \\
& 2016
\end{tabular}

Fonte: Silva Filho JP, et al., 2019.

\section{DISCUSSÃO}

Dentre os artigos analisados, entende-se que os pacientes críticos exibem ou estão na iminência de falência múltipla de órgãos, onde a capacidade de adaptação e a reserva fisiológica para alterações súbitas é praticamente inexistente, ficando suscetíveis a grandes instabilidades a menor das alterações, tendo como desfecho a deterioração clínica. Estas alterações, a terem lugar, têm um efeito multiplicativo no agravamento da circunstância clínica do doente, precisando na maioria das vezes de transferência para maca de transporte (GRAÇA ACG, et al., 2017). O transporte de pacientes críticos é procedimento 
complicado, que requer apropriada ponderação de riscos e benefícios, além do planejamento prévio para tornar mínimos os riscos.

Segundo Silva AM e Mendes FRP, et al. (2016), a segurança do paciente é a redução de riscos e prejuízos evitáveis durante o processo de cuidados à saúde a um mínimo oportuno, no entanto pesquisas e debates sinalizam que milhões de pacientes sofrem prejuízos incapacitantes, aumentando assim o tempo de permanência hospitalar ou mortes a cada ano, decorrentes de uma assistência de saúde insegura, necessitando geralmente de transferência para maca de transporte.

Conforme Meneguin S, et al. (2019), cada instituição hospitalar necessitam analisar a necessidade de ter uma equipe particularizada para realizar o transporte de pacientes, as proeminências na literatura de que 0 episódio de eventos adversos suaviza quando este recurso é usado, são escassas. O uso de sistemas de classificação do paciente, em relação à gravidade, pode ter aplicabilidade na prática clínica, mas a predição de risco relacionado ao transporte não é bem definida, pois alguns são inerentes ao próprio transporte e independem da distância e do tempo.

De acordo com Carneiro TA, et al. (2017), o transporte do paciente crítico representa um vasto desafio para os profissionais de saúde em especifico o enfermeiro por abranger a necessidade de integrar não apenas a tecnologia ao cuidado, mas, especialmente, o domínio de princípios científicos e, respectivamente, impõe-se o rigor de garantir as necessidades de tratamentos do paciente, tendo em vista minimizar os eventos adversos e garantir uma assistência segura e de qualidade ao paciente. Nessa vertente, considera-se essencial para a consolidação do conhecimento dos profissionais e a segurança ao paciente, o treinamento da equipe em ambientes simulados, que mimetizem uma condição real de transporte ao paciente crítico.

No entanto, a equipe de enfermagem necessita estar sempre habilitada para qualquer intercorrência durante o transporte, seja ele intra-hospitalar, ou extra-hospitalar. Nesse contexto as ações de enfermagem determinam a recuperação e/ou agravamento clínico dos pacientes. O cuidado de enfermagem é fundamental e é guiado por regras e rotinas que previnem os agravos à saúde (BOMFATI M, et al., 2019).

Dias CP, et al. (2019) relata que no que tange ao trabalho do enfermeiro, para desenvolver o cuidado de enfermagem, o profissional necessita ter o domínio do conhecimento técnico-científico, metodologias de pesquisa aplicadas à saúde, filosofias e políticas institucionais, além de toda a dimensão humanística indissociável ao ato de cuidar.

Pedreira LC, et al. (2014) corroborou em seu estudo que em relação às dificuldades encontradas no transporte do paciente crítico encontra-se também a ausência de comunicação anterior dos profissionais de enfermagem com o setor de destino desse paciente, sendo situação desfavorável para a realização apropriada do procedimento. As dificuldades de comunicação provocam esfinges e eventos adversos nos ambientes hospitalares e na realização de transporte do paciente crítico, também podendo acarretar em atrasos em seu encaminhamento, ou até mesmo na entrada destes na sala de exames, deixando-os expostos. Autores apontam ainda a comunicação como um dos pontos principais para um transporte essencial, tornando mínimo os erros e aumentando a segurança do paciente e equipe.

Os problemas de comunicação entre a unidade de origem e de destino têm sido apontados em diversos estudos como um dos principais fatores que colaboram para o episódio de eventos adversos durante o transporte de paciente crítico, como mostra estudo sobre a temática (MENEGUIN S, et al., 2019). Assim, a comunicação de informações concisas, organizadas e completas do paciente é uma responsabilidade extremamente importante para os profissionais de saúde, ou seja, sendo de essencial importância durante o planejamento do transporte, pois colabora para diminuir o período de espera para o exame, bem como, o tempo total gasto no procedimento em questão.

É importante ressaltar que para o acolhimento de pacientes críticos, o processo comunicativo necessita ser empregado como utensílio contínuo propiciadora de segurança e nitidez aos profissionais pelo fato de lidarem com circunstâncias estressantes que requerem atenção redobrada e perfeito entendimento das informações transmitidas (BALBINO AC e CARDOSO MVLML, 2017), uma vez que a comunicação anterior 
de todas as informações imprescindíveis entre a equipe que descola o paciente e também daquela que irá recebê-lo, de forma que não seja comprometida a sua segurança e continuidade dos cuidados de saúde seja reforçada.

O estudo realizado por Villois DF, et al. (2014) evidenciou que os eventos adversos foram registrados em $58,65 \%$ das transferências e apenas $47,11 \%$ das transferências foram realizada sob condições ideais de monitoramento e materiais necessários. Dessa forma, Veiga VC, et al. (2019), comenta que os eventos adversos estão relacionados a piores desfechos durante internações hospitalares. Melhores processos e capacitação profissional podem diminuir o episódio destes eventos e contribuir para um menor tempo médio de internação e os custos de hospitalização.

Para que aconteça um transporte garantido, é indispensável um bom planejamento, uma equipe habilitada e, quando necessário for, o uso de equipamentos confiáveis, para submissão de um paciente ao transporte, seja ele intra-hospitalar, ou extra-hospitalar (ZUCHELO LTS e CHIAVONE PA, 2009). Cabe salientar ainda que no transporte do doente crítico, por exemplo, o enfermeiro pode atuar na fase decisão, aconselhando ou não o transporte do doente. $\mathrm{Na}$ fase de planejamento, prestando os cuidados inerentes a sua profissão, tendo em vista a estabilização do doente. E, na fase de efetivação, vigiando o transporte para que não ocorra intercorrências.

Carneiro TA, et al (2017), ressaltaram em seu estudo que o enfermeiro mostrou participação ativa na maioria dos transportes realizados, entretanto, necessita ser assimilado que a oferta de maior segurança, tanto para o paciente quanto para o profissional, pode ser alcançada com o planejamento de estratégias assistenciais pelas equipes qualificadas para realizar o transporte intra-hospitalar e por meio da criação de protocolos operacionais padrão direcionados à demanda da unidade.

Ainda nesta mesma linha de considerações Hemesath MP, et al. (2017), afirma que a implantação de uma ferramenta organizada permitiu qualificar e padronizar os componentes de segurança a serem verificados antes, durante e após as transferências, beneficiando a compreensão dos cuidados imprescindíveis à segurança dos pacientes durante o transporte. Esta ferramenta contribui para qualificar $o$ ensino dos profissionais de enfermagem e promover estudos na área da transferência do cuidado.

É importante destacar que o enfermeiro vem acompanhando o aumento de tecnologias novas na área hospitalar e com isso vem aprimorando seus conhecimentos para executar seus cuidados junto à população hospitalizada. No entanto, faz-se ainda necessário aprimorar os cuidados de forma ampla no que se refere ao transporte de doente crítico, diminuindo os agravos à saúde da população durante este processo.

Portanto, faz-se necessário que haja uma padronização nas ações e fluxos através da elaboração de um Protocolo Institucional, juntamente com o Serviço da Educação Continuada, o qual tem papel fundamental na implementação de novos processos no dia-a-dia e treinamento dos profissionais, necessitam estar à disposição para minimização dos eventos adversos, obtendo assim uma assistência com excelência, mantendo a segurança do paciente.

\section{CONSIDERAÇÕES FINAIS}

Neste estudo ficou claro que em virtude da variedade da área de intervenções e fatores que intervêm e, em alguns casos, limitam as intervenções, o transporte do paciente crítico é uma circunstância complicada que envolve aspectos proeminentes, especialmente no que se refere à atuação dos profissionais durante o seu processo. Para o transporte do paciente crítico é indispensável que seja seguido uma série de passos. Sua realização necessita ser perpetrada de modo sólido e científico, assim como necessitam ser empregados conhecimentos teóricos e práticos e incorporados às novas tecnologias da área da saúde. Portanto, percebe-se que ações de melhorias para um transporte seguro de todos os pacientes críticos precisam ser criadas, padronizando ações e processos através de protocolos para que todos os profissionais sigam a mesma linha de cuidado. 


\section{REFERÊNCIAS}

1. ALMEIDA AC, et al. Transporte intra-hospitalar de pacientes adultos em estado crítico: complicações relacionadas à equipe, equipamentos e fatores fisiológicos. Acta Paul Enferm, São Paulo, 2012; 25(3): 471-6.

2. ANDRADE PB, et al. Impacto da transferência inter-hospitalar nos resultados da intervenção coronária percutânea primária. Rev. Bras. Cardiol. Intensiva, São Paulo, 2012; 20(4).

3. BALBINO AC, CARDOSO MVLML. Dificuldades no transporte inter-hospitalar de recém-nascido crítico realizado pelas equipes do serviço de atendimento móvel de urgência. Texto Contexto Enferm, 2017; 26(3): 1-8.

4. BOMFATI M, et al. Transporte intra/extra-hospitalar de crianças: implicações da equipe de enfermagem. Rev. Espaço para saúde, 2019; 20(1): 40-47.

5. CARNEIRO TA, et al. Critical patient transport: a challenge for the 21st century. J Nurs UFPE on line.,2017; 11(1): 70-6.

6. DIAS CP, et al. A importância do trabalho em equipe no transporte aéreo de pacientes. Rev enferm UFPE online. 2017; 11(6): 2408-14.

7. FRANÇOLIN L, et al. Gerenciamento da segurança do paciente sob a ótica dos enfermeiros. Rev. Esc. Enferm. USP, São Paulo, 2015; 49(2): 277-283.

8. GRAÇA ACG, et al. Transporte inter-hospitalar do doente crítico: a realidade de um hospital do nordeste de Portugal. Revista de Enfermagem Referência, 2017; 5(6): 133-144.

9. HEMESATH MP, et al. Comunicação eficaz nas transferências temporárias do cuidado de pacientes hospitalizados. Rev Gaúcha Enferm., 2019; 40(esp): 1-6.

10. MENDES KDS, et al. Revisão integrativa: método de pesquisa para incorporação de evidências na saúde e na enfermagem. Rev. Texto Contexto Enferm., Florianópolis, 2008; 17(4): 758-64.

11. MENEGUIN S, et al. Caracterização do transporte de pacientes críticos na modalidade intra-hospitalar. Acta Paul. Enferm., São Paulo, 2014; 27(2): 115-9.

12. MORAIS SA, ALMEIDA LF. Por uma rotina no transporte intra-hospitalar: elementos fundamentais para a segurança do paciente crítico. Revista Hupe, Rio de Janeiro, 2013; 12(3): 138-146.

13. ONG MS, COIERA E. Safety through redundancy: a case study of in-hospital patient transfers. Qual Saf Health Care, 2010; 19(5).

14. PEDREIRA LC, et al. Conhecimento da enfermeira sobre o transporte intra-hospitalar do paciente crítico. Rev. Enferm. UERJ, Rio de Janeiro, 2014; 22(4): 533-9.

15. SILVA AO, MENDES FRP. Importância De Parcerias em Pesquisas e Eventos Internacionais Para O Crescimento Da Ciência. Revista Ciência Plural, 2017; 3: 1-74.

16. VEIGA VC, et al. Adverse events during intrahospital transport of critically ill patients in a large hospital. Rev Bras Ter Intensiva, 2019; 31(1): 15-20.

17. VILLOIS DF, et al. Seguridad en el traslado intrahospitalario de pacientes criticos en pediatria. Medicina Infantil, 2014; 21 (esp): 237-243.

18. ZUCHELO LTS, CHIAVONE PA. Transporte intra-hospitalar de pacientes sob ventilação invasiva: repercussões cardiorrespiratórias e eventos adversos. Jornal Brasileiro de Pneumologia, 2009; 35(4): 367-374. 